\title{
Some Complexity Results for SystemVerilog Assertions
}

\author{
Doron Bustan and John Havlicek \\ Freescale Semiconductor, Inc.
}

\begin{abstract}
SystemVerilog Assertions (SVA) is a linear temporal logic within the recently approved IEEE 1800 SystemVerilog standard. The complexities of the satisfiability and model-checking problems are studied for a basic subset of SVA and for extensions of the basic subset obtained by adding each of the following features: local variables, regular expression intersection, quantified variables, and property declarations with arguments. It is shown that the complexities for the basic subset are PSPACE-complete, while the complexities increase to EXPSPACEcomplete 1 in each of the extensions. Alternating Büchi automata constructions provide the upper bounds, while reductions from PSPACE and EXPSPACE tiling problems provide the lower bounds.
\end{abstract}

\section{Introduction}

SystemVerilog Assertions, abbreviated SVA, is the assertion sublanguage of the recently approved IEEE 1800 SystemVerilog standard [14]. It is a linear temporal logic that is intended to be used to represent correctness properties and functional coverage events for the validation and verification of SystemVerilog designs. Prior to revision in the IEEE P1800 committee, development of SystemVerilog was carried out within the Accellera Organization, culminating in Accellera SystemVerilog 3.1a 1]. Industrial interest in SVA has been growing, as evidenced by its support in electronic design automation (EDA) tools, its discussion among verification engineers [19, and its deployment by semiconductor companies.

SVA has been developed in parallel with another recently approved standard assertion language, IEEE 1850 Property Specification Language (PSL) [15]. The two languages share a common core based on regular expressions. The common core includes temporal properties built using implication with regular expression antecedent and the standard logical boolean operators. While SVA and PSL have syntactic differences, there has been substantial work within Accellera and IEEE committees to ensure that the languages are aligned on the semantics of the common core. The two languages differ more substantially outside the common core.

${ }^{1}$ EXPCACE is defined us $\bigcup_{k \in \text { G }} \operatorname{DSPACE}\left(2^{n^{k}}\right)$. 
PSL provides the standard LTL operators, which are not in SVA, quantified variables, and numerous derived operators 2 PSL, similar languages, such as ForSpec, and academic simplifications have also received attention in recent literature 5496. However, there remain gaps in the analysis of the complexity of these languages for model checking. SVA, on the other hand, provides local variables as one of its distinguishing features. A local variable is used to capture the value of an expression at one point within a property and hold it for later reference, after which the local variable may be reassigned. Without local variables, complex auxiliary state machines are often required to represent temporal properties of practical interest. This makes local variables extremely useful for an industrially deployed temporal logic. The semantics of local variables has been studied in the committees [10, but SVA with local variables has received little attention in the literature and the expressiveness and complexity of the logic have not been studied before. This theoretical deficit has resulted in a certain amount of confusion in the industrial verification community. EDA companies disagree on what part of SVA should be supported in model checking, and verification engineers do not know whether properties that run in simulation can reasonably be expected to be supported in formal verification.

In this paper, we fill some of the theoretical gaps by studying the expressiveness and complexity of a basic subset of SVA and several of its extensions. The basic subset is obtained from the common core by eliminating the intersection operator on regular expressions. Previous work shows that the basic subset can express all omega-regular languages [4] and that the satisfiability and modelchecking problems for the basic subset are in PSPACE [78]. We prove a matching lower bound, hence these problems for the basic subset are PSPACE-complete.

We prove that extending the basic subset of SVA with either local variables or intersection of regular expressions increases the complexity of satisfiability and model-checking from PSPACE-complete to EXPSPACE-complete. For each of these extensions, we present a construction of alternating Büchi automata that gives an algorithm with tight complexity for the satisfiability and model-checking problems. These constructions also prove that the extensions do not increase expressiveness. Adding both local variables and intersection, the complexity of these problems in the combined extension remains EXSPACE-complete. We also study extension of the basic subset with quantified variables analogous to those in PSL. The satisfiability and model-checking problems for this extension are EXSPACE-complete. Finally, we study extension of the basic subset with declared properties and arguments. Their addition also results in EXPSPACEcomplete complexity for satisfiability and model checking. With the exception of those involving local variables, each of the results also holds for the corresponding subset of PSL.

Our lower bounds imply that, from the point of view of complexity classes, addition to the basic subset of any one of local variables, regular expression intersection, quantified variables, or declared properties with arguments is not harder

${ }^{2}$ PSL also offers a branching subset with CTL-like syntax and semantics. In this paper we consider only the linear subset of PSL. 
than another. Statements like "local variables cannot be used in model checking" and "local variables can be used in model checking only if they are restricted to analogues of PSL quantified variables" are not justified by our complexity analysis. The jump from PSPACE-hard to EXPSPACE-hard is significant, though, and model checking of arbitrary properties in these extensions is not generally considered practical. This does not mean that such features should not be supported in model-checking tools. Consider the simple use of integer constant parameters in operators, such as $[* n]$ (repeat $n$ times). This feature also makes the model-checking problem EXPSPACE-hard because integers have logarithmic representation [2]. We do not conclude that parameters should be written in unary or that declaration and instantiation of properties with arguments should be disallowed for formal verification. Such features are considered indispensable in industrially deployed languages. Similarly, local variables, regular expression intersection, and quantified variables are in the standardized assertion languages because they have proven to be very useful for writing properties in industrial practice. By understanding how the various language features contribute to the complexity, verification engineers can make more informed decisions about tradeoffs in coding styles for properties and can better estimate their tractability in formal verification. Tool builders also can better target the sources of hardness for model checking while providing a richer feature set in the property language that is supported. Hopefully, the automata constructions from this paper will provide a starting point for broader EDA tool support for formal verification using SVA.

\section{Preliminaries}

Given a set $A, A^{*}$ denotes the set of finite words over $A, A^{\omega}$ denotes the set of infinite words over $A$, and $A^{\infty}$ denotes the union $A^{*} \cup A^{\omega}$. The length of word $u \in A^{\infty}$ is denoted $|u|$. The empty word is denoted $\varepsilon$. The letters of $u$ are indicated by superscripts and are indexed consecutively beginning at zero. If $|u|>0$, then the first letter of $u$ is denoted $u^{0}$; if $|u|>1$, then the second letter of $u$ is denoted $u^{1}$; and so forth.

SVA has four language layers: boolean, sequence, property, and statement. The boolean layer consists of boolean expressions in which each variable referenced is either a design variable or a local variable of the assertion. $\Sigma$ denotes the finite alphabet of valuations of the design variables. The sequence layer consists of regular expressions over the boolean layer. Every regular language of finite words over $\Sigma$ can be represented by a suitably chosen sequence. The property layer combines sequences to create temporal logic formulas. The statement layer defines whether a property is to be evaluated as an obligation, an assumption, or a coverage goal. SVA statements are not discussed further in this paper.

For simplicity, we restrict each local variable to have a single-bit, boolean type (i.e., type bit in SystemVerilog). For a given finite set of sequences and properties, there is a finite set $V$ of local variables that appear therein. The set of valuations of these local variables is $2^{V}$. It is understood that $V$ is disjoint from 
the set of design variables. The set of semantic equivalence classes of boolean expressions can be identified with $2^{\Sigma \times 2^{V}}$. The constant "true" is denoted 1, and the constant "false" is denoted 0 .

For the rest of this document we use the following notations: $\sigma$ denotes a letter in $\Sigma ; w, x, y, z$ denote finite or infinite words over $\Sigma ; v$ denotes a one-bit local variable; $b$ and $e$ denote boolean expressions; $R$ denotes a sequence; $P$ denotes a property; and $L$ denotes a local variable valuation in $2^{V}$. Primes, subscripts, and superscripts are also added to these notations. The grammar for SVA sequences is

$$
\begin{aligned}
& R::=b|(1, v=e)|(R)|R \# \# 0 R| R \# \# 1 R \mid R \text { or } R \mid \\
& R \text { intersect } R|R[* 0]| R[* 1: \$]
\end{aligned}
$$

$R[* 0: \$]$ is an abbreviation for $R[* 0]$ or $R[* 1: \$]$, and, for $n>0, R[* n]$ is an abbreviation for the concatenation $R \# \# 1 \cdots \# \# 1 R(n$ copies of $R)$.

The grammar for SVA properties is

$$
P:=R|(P)| P \text { or } P \mid P \text { and } P|R|->P \mid \operatorname{not} P
$$

$R$ seq $P$ is an abbreviation for not $(R \mid->($ not $P$ )). The operator seq is the dual of $\mid->3$

Tight Satisfaction of Sequences. Tight satisfaction is a four-way relation, denoted $w, L_{0}, L_{1} \equiv R$, that defines when a finite word $w$ together with input local variable valuation $L_{0}$ satisfies a sequence $R$ and yields output local variable valuation $L_{1}$. Determination of whether the relation holds can be thought of as evaluation of $R$ over $w$ starting with local variable valuation $L_{0}$. As $R$ is evaluated, its local variables may be assigned, reassigned, and referenced at various points. In order for the result of the evaluation to be well-defined, a reference to a local variable must not be made unless the structure of $R$ ensures that the local variable holds a well-defined value at that point. There is some subtelty to this requirement. For example, a local variable may be assigned inconsistently in the two operands of intersect, after which a well-defined value cannot be guaranteed.

The IEEE 1800 standard addresses this problem by restricting syntactically the places within a sequence or property at which a given local variable can be referenced 4 These restrictions ensure that references to local variables yield welldefined values [10]. For example, if a local variable is assigned in both operands of an intersect, then the local variable cannot be referenced after the intersect until it has been reassigned a well-defined value. We assume that all top-level properties satisfy the restrictions (e.g., as checked by a compiler). This allows us to simplify the definition of tight satisfaction from that given in 14 .

For a boolean expression $b$ and $(\sigma, L) \in \Sigma \times 2^{V}$, let $b[\sigma, L]$ denote the boolean value obtained by evaluating the expression $b$ using the valuation $(\sigma, L)$ of the design and local variables. The tight satisfaction relation is defined as follows:

\footnotetext{
3 "seq" is not an explicit operator of SVA. It is equivalent to the the follows_by operator of ForSpec 4 .

${ }^{4}$ See the recursive functions flow, sample, and block defined in Annex E of 14 .
} 
- $w, L_{0}, L_{1} \equiv b$ iff $|w|=1$ and $b\left[w^{0}, L_{0}\right]=1$ and $L_{1}=L_{0}$.

$-w, L_{0}, L_{1} \equiv(1, v=e)$ iff $|w|=1$ and $L_{1}$ results from $L_{0}$ by assigning $e\left[w^{0}, L_{0}\right]$ to $v$.

$-w, L_{0}, L_{1} \equiv(R)$ iff $w, L_{0}, L_{1} \equiv R$.

$-w, L_{0}, L_{1} \equiv R_{1} \# \# 0 R_{2}$ iff there exist $x, y, z, L^{\prime}$ such that $w=x y z$ and $|y|=1$ and $x y, L_{0}, L^{\prime} \equiv R_{1}$ and $y z, L^{\prime}, L_{1} \equiv R_{2}$.

$-w, L_{0}, L_{1} \equiv R_{1} \# \# 1 R_{2}$ iff there exist $x, y, L^{\prime}$ such that $w=x y$ and $x, L_{0}, L^{\prime} \equiv R_{1}$ and $y, L^{\prime}, L_{1} \equiv R_{2}$.

- $w, L_{0}, L_{1} \equiv R_{1}$ or $R_{2}$ iff either $w, L_{0}, L_{1} \equiv R_{1}$ or $w, L_{0}, L_{1} \equiv R_{2}$.

- $w, L_{0}, L_{1} \equiv R_{1}$ intersect $R_{2}$ iff there exist $L^{\prime}, L^{\prime \prime}$ such that $w, L_{0}, L^{\prime} \equiv R_{1}$ and $w, L_{0}, L^{\prime \prime} \equiv R_{2}$ and $L_{1}(v)=L^{\prime}(v)$ if $v$ is assigned in $R_{1}$, and $L_{1}(v)=$ $L^{\prime \prime}(v)$ otherwise.

$-w, L_{0}, L_{1} \equiv R[* 0]$ iff $|w|=0$ and $L_{1}=L_{0}$.

$-w, L_{0}, L_{1} \equiv R[* 1: \$]$ iff there exist $j \geq 1$ and $L_{(0)}=L_{0}, w_{1}, L_{(1)}, w_{2}, L_{(2)}$, $\ldots, w_{j}, L_{(j)}=L_{1}$ such that $w=w_{1} w_{2} \cdots w_{j}$ and for every $i$ such that $1 \leq i \leq j, w_{i}, L_{(i-1)}, L_{(i)} \equiv R$.

The asymmetry of the definition of $L_{1}$ in the case of intersect is justified as follows. If $v$ is assigned in both $R_{1}$ and $R_{2}$, then the syntactic restrictions bar its reference after the intersect until it is reassigned. Therefore, we are free to let $v$ take either the value from $L^{\prime}$ or the value from $L^{\prime \prime}$.

Satisfaction of Properties. Satisfaction is a three-way relation, denoted $w, L \models P$, that defines when an infinite word $w$ together with input local variable valuation $L$ satisfies a property $P$

$-w, L \models R$ iff there exist $x, y, L^{\prime}$ such that $w=x y,|x|>0$, and $x, L, L^{\prime} \equiv R$.

$-w, L \models(P)$ iff $w, L \models P$.

- $w, L \models P_{1}$ or $P_{2}$ iff either $w, L \models P_{1}$ or $w, L \models P_{2}$.

- $w, L \models P_{1}$ and $P_{2}$ iff both $w, L \models P_{1}$ and $w, L \models P_{2}$.

$-w, L \models R \mid \rightarrow P$ iff for all $x, y, z, L^{\prime}$ such that $w=x y z$ and $|y|=1$ and $x y, L, L^{\prime} \equiv R, y z, L^{\prime} \models P$.

$-w, L \models \operatorname{not} P$ iff $w, L \not \models P$.

Let $P$ be a top-level property. The syntactic restrictions on references to local variables guarantee that for any $L, L^{\prime}, w, L \models P$ iff $w, L^{\prime} \models P$. We write $w \models P$ iff for some (equivalently, for all) $L \in 2^{V}, w, L \models P$, and we let $\mathcal{L}(P)$ denote the set $\left\{w \in \Sigma^{\omega} \mid w \models P\right\}$. A model satisfies $P$ iff each of its infinite computation traces satisfies $P$.

An SVA property is in positive normal form (PNF) if it does not contain any not operator. Positive normal form can be achieved by using DeMorgan's laws and the duality of $\mid->$ and seq to push all not operators down until they apply only to the boolean layer, where they can be absorbed into the boolean expressions. The case of not $R$ is handled by using the fact that, as property, $R$ is equivalent to $R$ seq 1 . 
Automata. A nondeterministic finite word automaton $(N F W)$ is a tuple $N=$ $\left\langle\Sigma, S, S_{0}, \rho, F\right\rangle$, where $\Sigma$ is a finite alphabet, $S$ is a finite set of states, $\rho \subseteq$ $S \times \Sigma \times S$ is a transition relation $5 S_{0} \subseteq S$ is a set of initial states, and $F \subseteq S$ is a set of accepting states. A sequence $\xi=\xi^{0} \xi^{1} \cdots \xi^{k} \in S^{*}(k \geq 0)$ is a run of $N$ over the finite word $w \in \Sigma^{*}$ provided $k=|w|, \xi^{0} \in S_{0}$, and for every $0 \leq i<|w|$, $\left(\xi^{i}, w^{i}, \xi^{i+1}\right) \in \rho$. The run $\xi$ is accepting if $\xi^{|w|} \in F$. An NFW $N$ accepts a word $w$ if there exists an accepting run of $N$ over $w$. We use $\mathcal{L}(N)$ to denote the set of words in $\Sigma^{*}$ that are accepted by $N$.

A nondeterministic Büchi automaton $(N B W)$ is a tuple $\left\langle\Sigma, S, S_{0}, \rho, F\right\rangle$ defined similarly to NFW. The automaton accepts an infinite word $w$ iff it has an infinite run over $w$ which contains infinitely many accepting states.

For a given set $X$, let Bool $^{+}(X)$ be the set of positive Boolean formulas over $X$ (i.e., Boolean formulas built from elements in $X$ using $\wedge$ and $\vee$ ), where we also allow the formulas true and false. Let $Y \subseteq X$. We say that $Y$ satisfies a formula $\theta \in \mathrm{Bool}^{+}(X)$ if the truth assignment that assigns true to the members of $Y$ and assigns false to the members of $X \backslash Y$ satisfies $\theta$. A tree is a prefix-closed subset $X \subseteq \mathbb{N}^{*}$.

An alternating Büchi word automaton $(A B W)$ is a tuple $B=\left\langle\Sigma, Z, z_{0}, \delta, A\right\rangle$, where $\Sigma, Z$, and $A$ are as $\Sigma, S$, and $F$ (respectively) in the definition of NFW, $z_{0} \in Z$ is a single initial state, and $\delta: Z \times \Sigma \rightarrow \mathrm{Bool}^{+}(Z)$ is a transition function. A run tree of $B$ on an infinite word $w \in \Sigma^{\omega}$ is a pair $(X, \tau)$ where $X$ is a (possibly infinite) tree and $\tau: X \rightarrow Z$ is a labeling function such that $\tau(\varepsilon)=z_{0}$ and such that the following holds: if $x \in X,|x|=i, \tau(x)=z$, and $\delta\left(z, w^{i}\right)=\theta$, then $x$ has $k$ children $x_{1}, \ldots, x_{k}$ in $X$ for some $0 \leq k \leq|Z|$ and $\left\{\tau\left(x_{1}\right), \ldots, \tau\left(x_{k}\right)\right\}$ satisfies $\theta$. The run tree $(X, \tau)$ is accepting if every infinite branch in $X$ has infinitely many labels in $A$. Note that the run tree can also have finite branches: if $|x|=i$, $\tau(x)=z$, and $\delta\left(z, w^{i}\right)=$ true, then $x$ need not have any children. $B$ accepts a word $w \in \Sigma^{\omega}$ if there exists an accepting run tree of $B$ over $w$. We use $\mathcal{L}(B)$ to denote the set of words in $\Sigma^{\omega}$ that are accepted by $B$.

An alternating transition system $(A T S)$ is a tuple $B=\left\langle\Sigma, Z, Z_{0}, \delta, A\right\rangle$, where $\Sigma, Z, \delta$, and $A$ are as in the definition of ABW and $Z_{0} \subseteq Z$ is a set of initial states. For $z \in Z_{0}, B^{(z)}$ is the ABW that results from $B$ by replacing $Z_{0}$ by $z$.

SVA Subsets. The basic subset of SVA, denoted SVA ${ }^{b}$, is obtained by limiting the sequence operators to \#\#1, \#\#0, or, $[* 0]$, and $[* 1: \$]$. All SVA property operators are allowed, but local variables and intersect are excluded. Every operator in $\mathrm{SVA}^{b}$ has an equivalent operator in PSL. We consider the following extensions to the basic subset: (1) $\mathrm{SVA}^{b+l}$, obtained by adding local variables; (2) SVA $^{b+i}$, obtained by adding the intersect operator; (3) SVA ${ }^{b+d}$, obtained by adding declared properties with arguments; and (4) SVA ${ }^{b+q}$, obtained by adding quantified variables analogous to those in PSL6 SVA $^{b+l+i}$ denotes the extension of $\mathrm{SVA}^{b}$ by adding both local variables and the intersect operator. The quantified variables feature is defined as follows. Let $P$ be a property with

${ }^{5} \rho$ can also be given as a function $S \times \Sigma \rightarrow 2^{S}$.

${ }^{6}$ SVA does not have quantified variables, so SVA ${ }^{b+q}$ is not, strictly speaking, a subset of SVA. However, SVA ${ }^{b+q}$ is equivalent to a subset of PSL. 
free variable $x$. Then "for $x$ in $S$ : and $P$ " is equivalent to "and $\left.\cos _{s \in S} P\right|_{x \leftarrow s}$ " and "for $x$ in $S$ : or $P$ " is equivalent to "or $\left.s \in S P\right|_{x \leftarrow s}$ ". $\left.P\right|_{x \leftarrow s}$ results from $P$ by replacing every occurrence of $x$ by $s$.

\section{Automata Constructions for SVA}

In [7] it is shown how to construct an ABW for each formula from a subset of PSL. The size of the ABW is linear in the size of the formula. Every operator in $\mathrm{SVA}^{b}$ has an equivalent operator in this subset of PSL. Thus, for every property $P$ in $\mathrm{SVA}^{b}$ there exists an ABW with size linear in the size of $P$ that accepts $\mathcal{L}(P)$. For every property $P$ in $\mathrm{SVA}^{b+q}$ or in $\mathrm{SVA}^{b+d}$ there is a simple exponential translation to a property in $\mathrm{SVA}^{b}$. Therefore, there exists an ABW with size exponential in the size of $P$ that accepts $\mathcal{L}(P)$.

The construction presented in [7] first builds particular NFWs for the sequences and then uses these NFWs to construct the ABW. The construction of the $\mathrm{ABW}$ can take any NFW in place of the particular ones given for sequences, and the size of the ABW is linear in the sizes of the NFWs plus the number of property operators. While the PSL subset considered in [7] does not include intersection, an exponential construction for NFWs for regular expressions with the intersect operator is given in [13. Using this construction, it follows that for every property $P$ in SVA $^{b+i}$ there exists an ABW with size exponential in the size of $P$ that accepts $\mathcal{L}(P)$. In the rest of this section we present exponential ABW constructions for the SVA ${ }^{b+l}$ and SVA ${ }^{b+l+i}$ subsets.

NFW for Sequences with Local Variables. Let $R$ be a sequence in SVA ${ }^{b+l}$. We present an inductive construction of an NFW $N(R)$ for $R$. The construction will arrange a function $\lambda$ mapping the set of states of $N(R)$ to $2^{V}$. The base cases are as follows.

$-N(b)=\left\langle\Sigma,\{0,1\} \times 2^{V},\{0\} \times 2^{V}, \rho,\{1\} \times 2^{V}\right\rangle$, where $\rho$ is the set of $((0, L), \sigma$, $(1, L))$ such that $b[\sigma, L]=1 . \lambda((0, L))=\lambda((1, L))=L$.

$-N(R[* 0])=\left\langle\Sigma,\{0\} \times 2^{V},\{0\} \times 2^{V}, \emptyset,\{0\} \times 2^{V}\right\rangle . \lambda((0, L))=L$.

$-N((1, v=e))=\left\langle\Sigma,\{0,1\} \times 2^{V},\{0\} \times 2^{V}, \rho,\{1\} \times 2^{V}\right\rangle$, where $\rho$ is the set of $\left((0, L), \sigma,\left(1, L^{\prime}\right)\right)$ such that $L^{\prime}$ results from $L$ by assigning $e[\sigma, L]$ to $v$. $\lambda((0, L))=\lambda((1, L))=L$.

For the inductive cases, assume that we have constructed NFWs $N\left(R_{1}\right)=$ $\left\langle\Sigma, S_{1}, I_{1}, \rho_{1}, F_{1}\right\rangle$ and $N\left(R_{2}\right)=\left\langle\Sigma, S_{2}, I_{2}, \rho_{2}, F_{2}\right\rangle$ and associated functions $\lambda_{1}, \lambda_{2}$. The sets $S_{1}$ and $S_{2}$ are assumed to have been made disjoint.

$-N\left(\left(R_{1}\right)\right)=N\left(R_{1}\right) \cdot \lambda=\lambda_{1}$.

- $N\left(R_{1} \# \# 0 R_{2}\right)=\left\langle\Sigma, S_{1} \cup S_{2}, I_{1}, \rho_{1} \cup \rho_{2} \cup \rho, F_{2}\right\rangle$, where $\rho$ is the set of $\left(s_{1}, \sigma, s_{2}\right)$ such that there exist $s^{\prime} \in F_{1}$ and $s^{\prime \prime} \in I_{2}$ such that $\left(s_{1}, \sigma, s^{\prime}\right) \in \rho_{1}$ and $\left(s^{\prime \prime}, \sigma, s_{2}\right) \in \rho_{2}$ and $\lambda_{1}\left(s^{\prime}\right)=\lambda_{2}\left(s^{\prime \prime}\right) . \lambda=\lambda_{1} \cup \lambda_{2}$.

- N $\left(R_{1} \# \# 1 R_{2}\right)=\left\langle\Sigma, S_{1} \cup S_{2}, I, \rho_{1} \cup \rho_{2} \cup \rho, F_{2}\right\rangle$, where $I=I_{1} \cup I_{2}$ if $I_{1} \cap F_{1} \neq \emptyset$, $I=I_{1}$ otherwise, and $\rho$ is the set of $\left(s_{1}, \sigma, s_{2}\right)$ such that $s_{2} \in I_{2}$ and there exists $s^{\prime} \in F_{1}$ such that $\left(s_{1}, \sigma, s^{\prime}\right) \in \rho_{1}$ and $\lambda_{1}\left(s^{\prime}\right)=\lambda_{2}\left(s_{2}\right) . \lambda=\lambda_{1} \cup \lambda_{2}$. 
- $N\left(R_{1}\right.$ or $\left.R_{2}\right)=\left\langle\Sigma, S_{1} \cup S_{2}, I_{1} \cup I_{2}, \rho_{1} \cup \rho_{2}, F_{1} \cup F_{2}\right\rangle . \lambda=\lambda_{1} \cup \lambda_{2}$.

$-N\left(R_{1}[* 1: \$]\right)=\left\langle\Sigma, S_{1}, I_{1}, \rho_{1} \cup \rho, F_{1}\right\rangle$, where $\rho$ is the set of $\left(s, \sigma, s^{\prime}\right)$ such that $s \in F_{1}$ and there exists $s^{\prime \prime} \in I_{1}$ such that $\left(s^{\prime \prime}, \sigma, s^{\prime}\right) \in \rho_{1}$ and $\lambda_{1}(s)=\lambda_{1}\left(s^{\prime \prime}\right)$. $\lambda=\lambda_{1}$.

By $\left.N(R)\right|_{L}$ we mean the automaton obtained from $N(R)$ by eliminating from the set of initial states those that are not mapped by $\lambda$ to $L$.

Lemma 1. Let $R$ be a sequence in $\mathrm{SVA}^{b+l}$, and let $w \in \Sigma^{*} . w, L_{0}, L_{1} \equiv R$ iff $\left.N(R)\right|_{L_{0}}$ has an accepting run over $w$ that ends in a state mapped by $\lambda$ to $L_{1}$.

Lemma 2. Let $R$ be a sequence in $\mathrm{SVA}^{b+l}$, let $V$ be the set of local variables in $R$, and let $S(R)$ be the set of states in the NFW $N(R)$. Then $|S(R)|=O\left(|R| \cdot 2^{|V|}\right)$.

ABW for Properties with Local Variables. Let $P$ be a property in SVA S $^{b+l}$. We present an inductive construction of an ATS $B(P)$ for $P$, which extend the construction presented in [7]. The construction will arrange a function $\lambda$ mapping the set of states of $B(P)$ to $2^{V}$ in such a way that $\lambda$ induces a bijection when restricted to the set of initial states. For $L \in 2^{V},\left.B(P)\right|_{L}$ is the ABW that results from $B(P)$ by restricting to the single initial state that is mapped by $\lambda$ to $L$.

We assume that the properties are in PNF. Wherever a sequence $R$ other than 1 or 0 appears as a property, we understand it to be replaced by $R$ seq 1 . The base cases of the construction are as follows.

- B(1) $=\left\langle\Sigma,\{0\} \times 2^{V},\{0\} \times 2^{V}, \delta, \emptyset\right\rangle$, where $\delta((0, L), \sigma)=$ true. $\lambda((0, L))=L$.

- B(0) $=\left\langle\Sigma,\{0\} \times 2^{V},\{0\} \times 2^{V}, \delta, \emptyset\right\rangle$, where $\delta((0, L), \sigma)=$ false. $\lambda((0, L))=L$.

For the inductive cases, assume that we have constructed the NFW $N(R)=$ $\langle\Sigma, S, I, \rho, F\rangle$ and function $\lambda_{R}$ for sequence $R$ and, for $i=1,2$, the ATS $B_{i}=$ $\left\langle\Sigma, Z_{i}, Z_{i 0}, \delta_{i}, A_{i}\right\rangle$ and function $\lambda_{i}$ for property $P_{i}$. Let $\rho$ be given as a function $2^{S} \times \Sigma \rightarrow 2^{S}$, where $\rho\left(S^{\prime}, \sigma\right)=\cup_{s \in S^{\prime}} \rho(s, \sigma)$ for $S^{\prime} \subseteq S$. Assume also that the state sets $S, Z_{1}, Z_{2}$ have been made disjoint and are disjoint from $\{0\} \times 2^{V}$. Let $\left.I\right|_{L}=I \cap \lambda_{R}^{-1}(L)$ and, for $i=1,2$, let $z_{i 0, L}$ be the unique state of $Z_{i 0}$ mapped by $\lambda_{i}$ to $L$.

$-B\left(\left(P_{1}\right)\right)=B\left(P_{1}\right) \cdot \lambda=\lambda_{1}$.

$-B\left(R \mid \rightarrow P_{1}\right)=\left\langle\Sigma,\left(\{0\} \times 2^{V}\right) \cup S \cup Z_{1},\{0\} \times 2^{V}, \delta \cup \delta_{1}, S \cup A_{1}\right\rangle$, where $\delta$ is defined over $\left(\left(\{0\} \times 2^{V}\right) \cup S\right) \times \Sigma$ as follows.

- $\delta((0, L), \sigma)=\bigwedge \rho\left(\left.I\right|_{L}, \sigma\right) \wedge \bigwedge \delta_{1}(Z, \sigma)$, where $Z$ is the set of $z \in Z_{10}$ such that $\lambda_{1}(z) \in \lambda_{R}\left(\rho\left(\left.I\right|_{L}, \sigma\right) \cap F\right)$.

- $\delta(s, \sigma)=\bigwedge \rho(s, \sigma) \wedge \bigwedge \delta_{1}(Z, \sigma)$, where $Z$ is the set of $z \in Z_{10}$ such that $\lambda_{1}(z) \in \lambda_{R}(\rho(s, \sigma) \cap F)$.

$\lambda=\lambda^{\prime} \cup \lambda_{R} \cup \lambda_{1}$, where $\lambda^{\prime}$ maps $\{0\} \times 2^{V}$ by $((0, L)) \mapsto L$.

$-B\left(R\right.$ seq $\left.P_{1}\right)=\left\langle\Sigma,\left(\{0\} \times 2^{V}\right) \cup S \cup Z_{1},\{0\} \times 2^{V}, \delta \cup \delta_{1}, A_{1}\right\rangle$, where $\delta$ is defined over $\left(\left(\{0\} \times 2^{V}\right) \cup S\right) \times \Sigma$ as follows.

- $\delta((0, L), \sigma)=\bigvee \rho\left(\left.I\right|_{L}, \sigma\right) \vee \bigvee \delta_{1}(Z, \sigma)$, where $Z$ is the set of $z \in Z_{10}$ such that $\lambda_{1}(z) \in \lambda_{R}\left(\rho\left(\left.I\right|_{L}, \sigma\right) \cap F\right)$.

- $\delta(s, \sigma)=\bigvee \rho(s, \sigma) \vee \bigvee \delta_{1}(Z, \sigma)$, where $Z$ is the set of $z \in Z_{10}$ such that $\lambda_{1}(z) \in \lambda_{R}(\rho(s, \sigma) \cap F)$. 
$\lambda=\lambda^{\prime} \cup \lambda_{R} \cup \lambda_{1}$, where $\lambda^{\prime}$ maps $\{0\} \times 2^{V}$ by $((0, L)) \mapsto L$.

- $B\left(P_{1}\right.$ or $\left.P_{2}\right)=\left\langle\Sigma,\left(\{0\} \times 2^{V}\right) \cup Z_{1} \cup Z_{2},\{0\} \times 2^{V}, \delta \cup \delta_{1} \cup \delta_{2}, A_{1} \cup A_{2}\right\rangle$, where $\delta$ is defined over $\left(\{0\} \times 2^{V}\right) \times \Sigma$ by $\delta((0, L), \sigma)=\delta_{1}\left(z_{10, L}, \sigma\right) \vee \delta_{2}\left(z_{20, L}, \sigma\right)$. $\lambda=\lambda^{\prime} \cup \lambda_{1} \cup \lambda_{2}$, where $\lambda^{\prime}$ maps $\{0\} \times 2^{V}$ by $((0, L)) \mapsto L$.

- $B\left(P_{1}\right.$ and $\left.P_{2}\right)=\left\langle\Sigma,\left(\{0\} \times 2^{V}\right) \cup Z_{1} \cup Z_{2},\{0\} \times 2^{V}, \delta \cup \delta_{1} \cup \delta_{2}, A_{1} \cup A_{2}\right\rangle$, where $\delta$ is defined over $\left(\{0\} \times 2^{V}\right) \times \Sigma$ by $\delta((0, L), \sigma)=\delta_{1}\left(z_{10, L}, \sigma\right) \wedge \delta_{2}\left(z_{20, L}, \sigma\right)$. $\lambda=\lambda^{\prime} \cup \lambda_{1} \cup \lambda_{2}$, where $\lambda^{\prime}$ maps $\{0\} \times 2^{V}$ by $((0, L)) \mapsto L$.

Lemma 3. Let $P$ be a property in $\mathrm{SVA}^{b+l}$ in $P N F$, and let $w \in \Sigma^{\omega} \cdot w, L \models P$ iff $w \in \mathcal{L}\left(\left.B(P)\right|_{L}\right)$.

Lemma 4. Let $P$ be a property in $\mathrm{SVA}^{b+l}$ in $P N F$, let $V$ be the set of local variables in $P$, and let $Z(P)$ be the set of states in the ATS $B(P)$. Then $|Z(P)|=$ $O\left(|P| \cdot 2^{|V|}\right)$.

The Intersect Operator. In this section we extend the construction of ABWs for properties in $\mathrm{SVA}^{b+l}$ to SVA ${ }^{b+l+i}$. Assume that for the sequences $R_{1}$ and $R_{2}$ we have constructed the NFWs $N_{1}=\left\langle\Sigma, S_{1}, I_{1}, \rho_{1}, F_{1}\right\rangle$ with mapping $\lambda_{1}: S_{1} \rightarrow$ $2^{V}$ and $N_{2}=\left\langle\Sigma, S_{2}, I_{2}, \rho_{2}, F_{2}\right\rangle$ with mapping $\lambda_{2}: S_{2} \rightarrow 2^{V}$, respectively. We define $N\left(R_{1}\right.$ intersect $\left.R_{2}\right)=\left\langle\Sigma, S_{1} \times S_{2}, I, \rho, F_{1} \times F_{2}\right\rangle$, where $I=\left\{\left(i_{1}, i_{2}\right) \in\right.$ $\left.I_{1} \times I_{2} \mid \lambda_{1}\left(i_{1}\right)=\lambda_{2}\left(i_{2}\right)\right\}$ and

$$
\rho=\left\{\left(\left(s_{1}, s_{2}\right), \sigma,\left(s_{1}^{\prime}, s_{2}^{\prime}\right)\right) \mid\left(s_{1}, \sigma, s_{1}^{\prime}\right) \in \rho_{1} \text { and }\left(s_{2}, \sigma, s_{2}^{\prime}\right) \in \rho_{2}\right\}
$$

and we define $\lambda: S_{1} \times S_{2} \rightarrow 2^{V}$ by $\lambda\left(\left(s_{1}, s_{2}\right)\right)(v)=\lambda_{1}\left(s_{1}\right)(v)$ if $v$ is assigned in $R_{1}$ and $\lambda\left(\left(s_{1}, s_{2}\right)\right)(v)=\lambda_{2}\left(s_{2}\right)(v)$ otherwise. The following lemma extends the result of Lemma 1 to SVA ${ }^{b+l+i}$.

Lemma 5. Let $R$ be a sequence in $\mathrm{SVA}^{b+l+i}$, and let $w \in \Sigma^{*} . w, L_{0}, L_{1} \equiv R$ iff $\left.N(R)\right|_{L_{0}}$ has an accepting run over $w$ that ends in a state mapped by $\lambda$ to $L_{1}$.

Lemma 6. Let $R$ be a sequence in $\mathrm{SVA}^{b+l+i}$, let $V$ be the set of local variables in $R$, and let $S(R)$ be the set of states in the $N F W N(R)$. If $V$ is non-empty, then $|S(R)|=O\left(2^{|R| \cdot|V|}\right)$. Otherwise $|S(R)|=O\left(2^{|R|}\right)$.

Lemma 7. Let $P$ be a property in $\mathrm{SVA}^{b+l+i}$ in $P N F$, let $V$ be the set of local variables in $P$, and let $Z(P)$ be the set of states in the ATS $B(P)$. If $V$ is non-empty, then $|Z(P)|=O\left(2^{|P| \cdot|V|}\right)$. Otherwise $|Z(P)|=O\left(2^{|P|}\right)$.

Upper Bound Complexity. Following the automata-theoretic approach of [17, the satisfiability and model-checking problems for linear temporal logics are solved by representing the property by an ABW and then translating the ABW into an exponentially larger NBW 12. Satisfiability is solved by checking for emptiness of the NBW, and model-checking is solved by checking for emptiness of the product of the model with the NBW for the negation of the property. Since the emptiness problem for NBWs is in NLOGSPACE 18, the complexity of these problems is in PSPACE with respect to the number of states of the ABW [17. These observations and the automata constructions of the preceding sections lead to the following theorem. 


\section{Theorem 1}

- The satisfiability and model-checking problems for properties in any of the extensions $\mathrm{SVA}^{b+i}, \mathrm{SVA}^{b+q}$ SVA $^{b+d}$ are in EXPSPACE with respect to $|P|$.

- The satisfiability and model-checking problems for properties in $\mathrm{SVA}^{b+l}$ are in EXPSPACE with respect to $|V|$ and in PSPACE with respect to $|P|$.

- The satisfiability and model-checking problems for properties in $\mathrm{SVA}^{b+l+i}$ are in EXPSPACE with respect to $|V| \cdot|P|$.

\section{Lower Bounds}

In this section we explore some of the "sources of hardness" of SVA satisfiability and model-checking. In the previous section we showed that the satisfiability and model checking problems for properties in $\mathrm{SVA}^{b}$ are in PSPACE. A reduction from the PSPACE tiling problem [201116] can be used to prove the following.

Proposition 1. The satisfiability and model checking problems for properties in $\mathrm{SVA}^{b}$ are PSPACE-hard.

When SVA $^{b}$ is extended by local variables, regular expression intersection, declared properties with arguments, or quantified variables, the complexity of the model-checking and satisfiability problems become EXPSPACE-hard.

Theorem 2. The satisfiability and model checking problems for properties in $\mathrm{SVA}^{b+l}, \mathrm{SVA}^{b+i}, \mathrm{SVA}^{b+d}$, and SVA ${ }^{b+q}$ are EXPSPACE-hard.

We present a proof sketch by outlining a reduction from a version of the EXPSPACE tiling problem 201116] to each of the subsets. In the EXPSPACE tiling problem, the following are given: a finite set $T$ of "tiles", vertical and horizontal restrictive relations $\mathcal{V} \subseteq T \times T$ and $\mathcal{H} \subseteq T \times T$, an initial tile $\tau_{0} \in T$, a final tile $\tau_{a} \in T$, and an integer $n>0$. The problem is to decide whether there exists $m>0$ such that there exists a tiling of a $2^{n} \times m$ grid such that the following hold: (1) $\tau_{0}$ is in the bottom left corner; (2) the first occurrence of $\tau_{a}$ is in the top left corner; (3) every pair of horizontally neighboring tiles is in $\mathcal{H}$; and (4) every pair of vertically neighboring tiles is in $\mathcal{V}$.

Given an EXPSPACE tiling problem $\mathcal{T}=\left\langle T, \mathcal{V}, \mathcal{H}, \tau_{0}, \tau_{a}, n\right\rangle$, we define for each subset a property $P$, polynomia $\sqrt{7}$ in $n$, such that there exists an infinite word $w$ that satisfies $P$ iff there exists a tiling for $\mathcal{T}=\left\langle T, \mathcal{V}, \mathcal{H}, \tau_{0}, \tau_{a}, n\right\rangle$. The idea is to partition $w$ into substrings of length $n$ called "blocks" such that every block represents one tile. The $n$ letters in a block provide a binary encoding of a number between 0 and $2^{n}-1$, inclusive. We refer to this number as the counter value of the block. The blocks are enumerated in the order of increasing counter values modulo $2^{n}$, starting from 0 . Pairs of consecutive blocks (except those enumerated $\left.\left(2^{n}-1,0\right)\right)$ must satisfied $\mathcal{H}$, and pairs of blocks whose counter values are equal and are separated by $2^{n}-1$ intermediate blocks must satisfy $\mathcal{V}$.

${ }^{7}$ Quadratic for SVA ${ }^{b+i}$ and SVA ${ }^{b+d}$, linear for the other extensions. 
We construct the properties for the reductions to the different subsets in two parts. The shared part is common to all the properties and is in SVA ${ }^{b}$. For each subset we construct its specific part using the extending feature of the subset.

The alphabet for all properties is $2^{\{\boldsymbol{\alpha}, \diamond, c, z\}} \times T$, where $\boldsymbol{\phi}$ is a special signal that marks the beginning of a block, $\diamond$ is a special signal that is first high one letter after the last block of the tiling, $c$ is a one-bit signal used to represent the counter value of the block, and $z$ is an auxiliary variable used to represent the carry over when incrementing the counter value. We use the convention that the value of $c$ in the first (resp., last) letter of a block is the least (resp., most) significant bit of the counter value. The set $T$ of tiles is of constant size. The $T$ component of a letter is referenced by the signal $t$. The tile represented by a block is understood to be the value of $t$ in its last letter.

For lack of space, we do not present the shared part. It is obtained by creating properties in $\mathrm{SVA}^{b}$ of size $O(n)$ requiring that (1) a prefix of $w$ be partitioned into blocks of length $n$, the first letter of each being indicated by $\boldsymbol{\phi} ;(2)$ the counter values of the blocks increment modulo $2^{n}$ starting at $0 ;(3) \diamond$ appear for the first time after a block with counter value $2^{n}-1$ and mark the first letter after the end of the prefix; (4) the first block's tile value be $\tau_{0} ;(5)$ the tile value of the first block in the last row be $\tau_{a}$; and (6) every two consecutive blocks in the same row be in $\mathcal{H}$. It remains to construct for each of the extensions the specific part of the property requiring that a pair of blocks with the same counter value that are separated by $2^{n}-1$ intermediate blocks be in $\mathcal{V}$.

For the local variable extension, we let $R_{c \neq v}$ denote a sequence that is tightly satisfied by a block iff the counter value of the block is different than the binary value represented by the local variables $v_{0}, v_{1}, \ldots, v_{n-1}$. Such a sequence can be created with size $O(n)$. Also, we let $R_{c=v}$ denote the sequence

$c==v_{0} \# \# 1 \quad c==v_{1} \# \# 1 \cdots \quad \# \# 1 \quad c==v_{n-1}$,

which is tightly satisfied by a block iff the counter value of the block is equal to the binary value represent by $v_{0}, v_{1}, \ldots, v_{n-1}$. The specific part $P_{\mathcal{V}}^{l}$ for the local variable extension is as follows:

$$
\begin{aligned}
& \left(1[* \mathrm{n}] \# \# 0 \quad t !=\tau_{a}\right)[* 0: \$] \# \# 1 \\
& \left.\left.\left(1, v_{0}=c\right) \text { \#\#1 (1, } v_{1}=c\right) \text { \#\#1 } \cdots \text { \#\#1 (1, } v_{n-1}=c\right) \text { \#\#0 } t !=\tau_{a} \quad \mid-> \\
& \operatorname{and}_{\tau_{1} \in T} \quad\left(t==\left.\tau_{1} \quad\left|->1 \quad \# \# 1 \quad R_{c \neq v}[* 1: \$] \quad \# \# 1 \quad R_{c=v} \quad \# \# 0 \quad\right|\right|_{\mathcal{V}\left(\tau_{1}, \tau_{2}\right)} t==\tau_{2}\right)
\end{aligned}
$$

After any number of blocks with tile other than $\tau_{a}$, the counter value is stored in $v_{0}, v_{1}, \ldots, v_{n-1}$. Then a conjunction over $\tau_{1} \in T$ of implications whose antecedents test $t==\tau_{1}$ ensures that $\left(\tau_{1}, \tau_{2}\right) \in \mathcal{V}$, where $\tau_{2}$ is the tile of the next block at which the counter value equals the binary value saved in $v_{0}, v_{1}, \ldots, v_{n-1}$. The size of $P_{\mathcal{V}}^{l}$ is $O(n)$.

The specific part for the extension by quantified variables is similar:

$$
\begin{aligned}
& \text { for } v_{0}, \ldots, v_{n-1} \text { in bit: and } \\
& \left(1[* \mathrm{n}] \# \# \quad t !=\tau_{a}\right)[* 0: \$] \# \# 1 R_{c=v} \# \# 0 \quad t !=\tau_{a} \quad \mid-> \\
& \text { and }_{\tau_{1} \in T}\left(t==\tau_{1} \quad\left|->1 \quad \# \# 1 \quad R_{c \neq v}[* 1: \$] \# \# 1 \quad R_{c=v} \# \# 0\right|||_{\left(\tau_{1}, \tau_{2}\right)} t==\tau_{2}\right)
\end{aligned}
$$

Instances of properties with arguments can be used to universally quantify the arguments. In this way, we can adapt the specific part with quantified 
variables to serve as the specific part using declared properties with arguments. Here is an example to illustrate. Let $P(a, b, c)$ be a declared property with arguments. Define $P_{1}(b, c)=P(1, b, c)$ and $P(0, b, c), P_{2}(c)=P_{1}(1, c)$ and $P_{1}(0, c)$, $P_{3}=P_{2}(1)$ and $P_{2}(0)$. The property $P_{3}$ is equivalent to $\forall a \forall b \forall c: P(a, b, c)$. The adaptation involves $n$ declared properties, each of size $O(n)$, so the specific part is of size $O\left(n^{2}\right)$.

For the specific part using intersect, we assume that the following auxiliary sequences of size $O(n)$ have been constructed. For $0 \leq i \leq n-2, R_{i}$ is a sequence that is tightly satisfied by a series of two or more blocks provided the $i$ th bits of the counters of the first and last blocks are equal. $R_{n-1}$ is a sequence that is tightly satisfied by a series of two or more blocks provided the $(n-1)$ st bits of the counters of the first and last blocks are equal and, in addition, counter bit $n-1$ changes its value exactly twice in the intermediate blocks. The minimum number of intermediate blocks is $2^{n-1}$, and the maximum number is $3 \cdot 2^{n-1}+2$. Inside these boundaries, distinct blocks have distinct counter values. The sequence $R_{\text {tiles in }} \mathcal{V}$ is tightly satisfied by a series of blocks provided the pair formed by the tiles of the first and last blocks is in $\mathcal{V}$. From these auxiliary sequences, we construct $R_{\text {next }}=R_{0}$ intersect $\cdots$ intersect $R_{n-1}$, which is tightly satisfied by a series of blocks provided the first and last blocks have the same counter value and there are exactly $2^{n}-1$ intermediate blocks. $R_{\text {next }}$ is of size $O\left(n^{2}\right)$. The specific part $P_{\mathcal{V}}^{i}$ is as follows:

$$
\begin{aligned}
& \left(1[* n-1] \# \# 1 \quad t !=\tau_{a}\right)[* 0: \$] \# \# 1 \quad 1 \quad \mid-> \\
& \left(1[* n-1] \# \# 1 \quad t==\tau_{a}\right) \text { or }\left(R_{\text {next }} \text { intersect } R_{\text {tiles in }} \mathcal{V}\right)
\end{aligned}
$$

The consequent is tightly satisfied by a series of two or more blocks provided that either the tile of the first block is $\tau_{a}$ or the first and last blocks have the same counter value, their tiles are in $\mathcal{V}$, and there are exactly $2^{n}-1$ intermediate blocks. The property $P_{\mathcal{V}}^{i}$ requires the consequent for every block before $\tau_{a}$ occurs.

\section{Conclusions and Related Work}

There has been some prior work on the complexity of satisfiability and model checking of PSL and similar languages. In 4, the complexity of the ForSpec language is shown to be PSPACE-complete. In [3] there is a discussion of the complexity of the reset operator in Sugar and ForSpec. Symbolic verification of PSL properties restricted to negated sequences is explored in 6]. A construction of alternating Büchi automata for a large subset of PSL is presented in 7]. The automata from this construction run over both finite and infinite words, and the construction accounts for the neutral semantic variant for finite words.

In this paper we have explored the complexity of SVA. We have shown that when features like local variables, regular expression intersection, and declared properties with argument are used, the complexity of the language becomes EXPSPACE-complete. These results invite further investigation of the practical usage of these features and the performance of model-checking algorithms that support them. Other theoretical gaps in the complexity analysis remain. For 
example, the expressiveness and complexity of SVA with recursive properties and the complexity of checking SVA in simulation are still open.

\section{References}

1. Accellera. System Verilog 3.1a Language Reference Manual: Accellera's Extensions to Verilog. 2004. www.eda.org/sv/SystemVerilog_3.1a.pdf.

2. R. Alur and T. Henzinger. Real-time logics: complexity and expressiveness. In Proc. 5th Symp. on Logic in Computer Science, pages 390-401, June 1990.

3. R. Armoni, D. Bustan, O. Kupferman, and M. Y. Vardi. Resets vs. aborts in linear temporal logic. In Proc. 9th Int'l Conf. on Tools and Algorithms for the Construction and Analysis of Systems, volume 2619 of LNCS, pages 65-80, 2003.

4. R. Armoni, L. Fix, R. Gerth, B. Ginsburg, T. Kanza, A. Landver, S. Mador-Haim, A. Tiemeyer, E. Singerman, M.Y. Vardi, and Y. Zbar. The ForSpec temporal logic: A new temporal property-specification language. In Proc. 8th Int'l Conf. on Tools and Algorithms for the Construction and Analysis of Systems, volume 2280 of $L N C S$, pages 296-311, 2002.

5. I. Beer, S. Ben-David, C. Eisner, D. Fisman, A. Gringauze, and Y. Rodeh. The temporal logic Sugar. In Proc. 13th Int'l Conf. on Computer Aided Verification, volume 2102 of $L N C S$, pages 363-367, 2001.

6. S. Ben-David, D. Fisman, and S. Ruah. Embedding finite automata within regular expressions. In Int'l Symp. on Leveraging Applications of Formal Methods, 2004.

7. D. Bustan, D. Fisman, and J. Havlicek. Automata construction for PSL. Technical Report MCS05-04, The Weizmann Institute of Science, May 2005.

8. D. Bustan, A. Flaisher, O. Grumberg, O. Kupferman, and M.Y. Vardi. Regular vacuity. In 13th Advanced Research Working Conf. on Correct Hardware Design and Verification Methods, volume 3725 of LNCS, pages 191-206, 2005.

9. M. J. C. Gordon. Validating the PSL/Sugar semantics using automated reasoning. Formal Aspects of Computing, 15(4):406 - 421, December 2003.

10. J. Havlicek, K. Shultz, R. Armoni, S. Dudani, and E. Cerny. Notes on the semantics of local variables in Accellera SystemVerilog 3.1 concurrent assertions. Technical Report 2004.01, Accellera, May 2004. Available from www. accellera.org.

11. H.R. Lewis. Complexity of solvable cases of the decision problem for the predicate calculus. In Foundations of Computer Science, volume 19, pages 35-47, 1978.

12. S. Miyano and T. Hayashi. Alternating finite automata on $\omega$-words. Theoretical Computer Science, 32:321-330, 1984.

13. M.O. Rabin and D. Scott. Finite automata and their decision problems. IBM Journal of Research and Development, 3:115-125, 1959.

14. IEEE Computer Society. 1800: IEEE Standard for SystemVerilog-Unified Hardware Design, Specification, and Verification Language. IEEE, 2005.

15. IEEE Computer Society. 1850: IEEE Standard for Property Specification Language (PSL). IEEE, 2005.

16. P. van Emde Boas. The convenience of tilings. In Complexity, Logic and Recursion Theory, volume 187 of Lecture Notes in Pure and Applied Mathematics, pages 331363, 1997.

17. M.Y. Vardi. An automata-theoretic approach to linear temporal logic. In F. Moller and G. Birtwistle, editors, Logics for Concurrency: Structure versus Automata, volume 1043 of Lecture Notes in Computer Science, pages 238-266, 1996. 
18. M.Y. Vardi and P. Wolper. Reasoning about infinite computations. Information and Computation, 115(1):1-37, November 1994.

19. Verification guild: A community of verification professionals. http:// verificationguild.com.

20. H. Wang. Dominoes and the AEA case of the decision problem. In Symposium on the Mathematical Theory of Automata, pages 23-55, 1962. 